\title{
The Optical Network Design using Availability Models to Accept Additional Nodes
}

\author{
Jongboo Eun ${ }^{1, a}$, Gyoutae Park ${ }^{1, b}$, Gwanghoon Ahn ${ }^{1, c}$, and Hiesik Kim ${ }^{1, d}$ \\ ${ }^{1}$ Dept. of Electronics Engineering, University of Seoul, Korea \\ adata3000@nate.com, bgtparkgs@kgs.or.kr, 'ceiahn@gmail.com, ddrhskim@uos.ac.kr
}

Keywords : optical network, reliability, availability, traffic model

\begin{abstract}
It is so obvious that we have to consider factors of costs, the QoS (Quality of Service), and performance analysis for the design of an optical network. The models of Traffic and availability are significant factors for achieving the performance analysis from the initial step to deploying network. But current traffic models are solely used by a single segment. Whole networks are extended from a sing traffic model. On the other hand an availability model is just used to find the rate of hardware failures. In this paper, we implemented an availability model with regional conformance by inversely analyzing the definition of availability. Also, we computed the number of nodes and links to configure the logical topology of optical networks by using our designed availability. Furthermore, we proposed a method to design an optical network by using our novel availability model.
\end{abstract}

\section{Introduction}

The Queueing Theory cannot suggest the performance analysis model when the electrical telecommunication network is designed. Because it cannot consider the topography feature and uses the traffic assumed as the Erlang $B$ distribution [1]. Presently, the traffic model has been suggesting MMPP (Markov Modulated Poisson Process). But MMPP is insufficient to analyze the traffic of the multiple class type like the Internet. [2].

The important factors in the optical network design are cost, the QoS (Quality of Service), and the performance analysis. The modeling traffic and availability in the performance analysis of network are the significant factors. Also, those determine performance from the initial design stage to the construction of network. [3]. However, the current traffic models handle only the traffic of the single segment. On the other hand an availability model is just used to find the rate of hardware failures. [3, 4].

Generally the logical topology of an optical network describes the upper of an optical layer. The layer containing nodes in the network is the SONET/SDH layer. Synchronous optical networking is a method for communicating digital information using lasers or light-emitting diodes (LEDs) over optical fiber. The method was developed to replace the Plesiochronous Digital Hierarchy (PDH) system for transporting large amounts of telephone and data traffic and to allow for interoperability between equipment from different vendors. SDH or Synchronous Digital Hierarchy standard developed by the International Telecommunication Union (ITU), documented in standard G.707 and its extension G.708. The logical topology is more advantageous than physical one for traffics, routing, and stream control. In PSTN, as designing a network and analyzing the performance of network, we have been using only traffic models. In WDM, however, we consider the traffic and availability models for analyzing the performances of an optical network [5]. Also, Poisson distribution is inefficient in analyzing an optical network. So, recently, a new method is demanded to analyze the set of nodes and links to configure graphs of the electrical telecommunication network.

In this paper, we proposed a novel method to calculate a number of nodes and links of an optical network by inversely interpreting the definition of availability. Also, we computed the number of nodes and links to configure the logical topology of optical networks by using our designed availability. Furthermore, we proposed a method to design an optical network by using our novel availability model. 


\section{The Design of the optical network using the availability modeling}

Fig. 1 shows general structure of physical topology of an optical network. Table 1 shows failure rates of components to build an optical network like those of whole network. The unit of the rate of failure is FIT(failure in time). Here, $\mathrm{N}$ is a fiber number, $\mathrm{W}$ is a wavelength. Fig. 2 shows the logical topology for physical structure in an optical network.

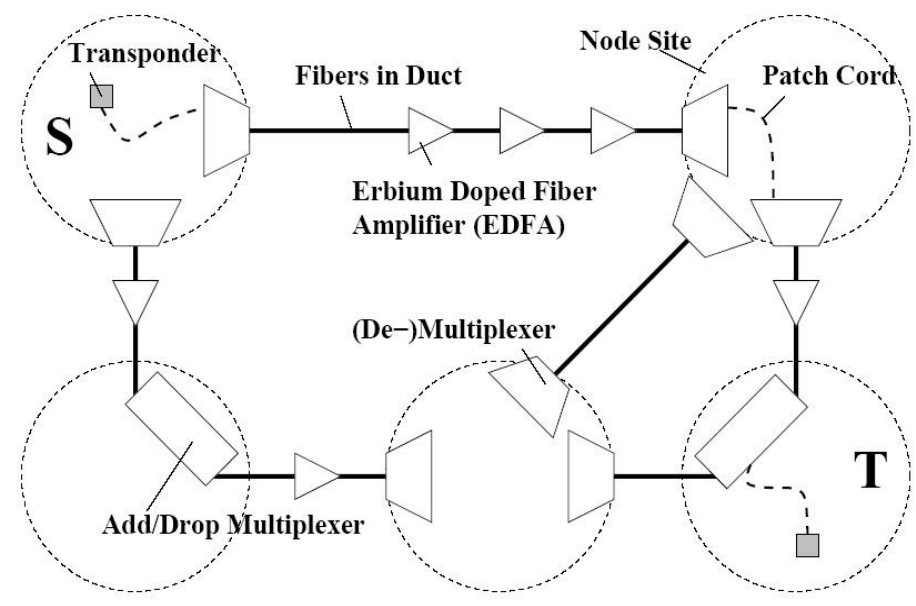

Fig. 1. Example of WDM network

Table 1 FIT for component of an optical network

\begin{tabular}{|c|c|}
\hline Components of network & Failure rates [FIT] \\
\hline \hline (De-)Multiplexer & $25 \times \mathrm{W}$ \\
EDFA & 2850 \\
Optical Switch 1 & $21 \mathrm{~W} \times \mathrm{W} / 4$ \\
Optical Switch 2 & $21 \times 2 \times 2 \times \mathrm{N}$ \\
Coupler 1 & $25 \times 2$ \\
Coupler 2 & $25 \times \mathrm{W} / 4$ \\
Coupler 3 & $25 \times(\mathrm{N}-1)$ \\
Tunable Transmitter & 745 \\
Fix Transmitter & 186 \\
Tunable Receiver & 470 \\
Fix Receiver & 70 \\
Digital Switch 1 & $875 \times \mathrm{W}$ \\
Digital Switch 2 & $875 \times \mathrm{W} \times \mathrm{N}$ \\
Wavelength Blocker & $50 \times \mathrm{W}$ \\
\hline
\end{tabular}

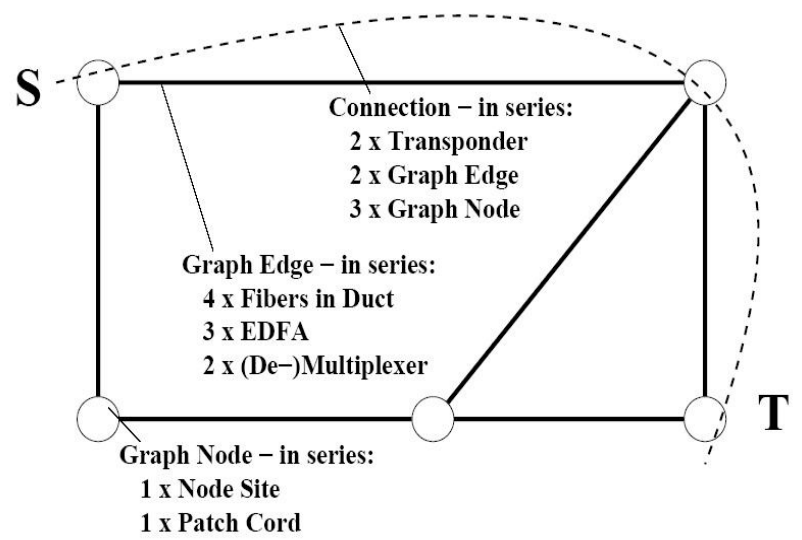

Fig. 2. Topology of an optical network

The link, connection, and availability about the network devices have to be altogether considered in order to handle the total availability of the optical network [5]. The link availability cannot distinguish whether the designed network is in the commerce area or the residential area. That is, the value quantitative of which is unable to reflect to flock the traffic according to the pertinent area feature is gotten. In conclusion, there are the many difference between the availabilities even though it is the link of the same length according to the topographical location. In the optical network design, it is unable to be properly reflected. In addition, the availabilities about the individual link in the designed area are altogether considered if it enlarges to the total network. In this paper, we solved the problem about the topographically location and proposed the availability model designing the optical network. 


\subsection{Definition of the availability model of the optical network}

The availability model enlarges it to the total network to calculate the availability with the connection between the specified nodes in which the communication is possible. Therefore, the availability model has to be suggested by considering each availability model about the resources (link, node, and connection) for comprising optical network. Also, the link becomes the available state if the duct or fiber can use. In conclusion, the availability model includes the duct availability, link, node defining the availability of many devices, and connection defining the availability of the path where it exists between the nodes when the availability model considering the topography is defined.

\subsection{Modeling of the link availability}

The duct availability and link availability are the items defining the availability of the cable. The availability about the duct among these can be shown with the Eq. 2.1.

$$
A_{\text {duot }}=\sum_{\text {Ank }}\left(c_{k} \cdot g_{k}\right)
$$

In the Eq. 2.2, $g_{k}$ is the real number selected in the range between 0 and 1 with the availability of the local $(k)$ where the duct $(g)$ is positioned. The $c_{k}$ is the real number selected in the range between 0 and 1 with the duct in the specific area $(k)$. And $\Sigma_{k} c_{k}$ equals 1 .

Also, the link availability is the element showing the availability of the cable. If the specific cable is the available state, existed all the OA (Optical Amplifier) and the regenerative repeater become the available state. In addition, the availability of the transponder has to be considered because the transponders are positioned in the both ends of the link in order to prepare the case where the malfunction is generated in the network. In short, the link availability can be presented like the Eq. 2.2 .

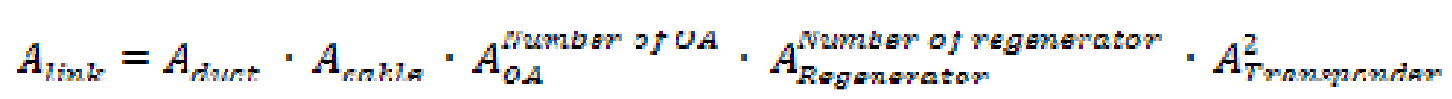

\subsection{Modeling of the node availability}

The node availability is determined according to the availability of all sorts of units comprising corresponding node. The unit comprising the node includes the topographical location of nodes, sub rack, optical unit, multiplex unit, switching unit, and etc. In brief, the node availability multiplies altogether by the availability of the single unit. They can be explained by the Eq. 2.3 .

$$
A_{\text {node }}=A_{\text {geo inmeinm }} \cdot A_{\text {subraek }} \cdot A_{\text {Cptigaln }_{n}} \cdot A_{M u X_{n}} \cdot A_{\text {switehing n }} \cdot A_{\text {add/drop n }}
$$

\subsection{Modeling of the connection availability}

If the source transponder and destination transponder can use all the network elements existing along the pathway, the relevant connection(i) becomes the available state. Also, if each node can use the other one's communication path, it can be said that 2 nodes are connected. The concept of this connection is applied even if this is expanded to the source transponder and destination transponder. 
If $\boldsymbol{A}_{\boldsymbol{j}}$ is assumed as the universal set of the network elements where the connection( $\left.\boldsymbol{i}\right)$ use currently the $\boldsymbol{S}_{i}$ among the network element as the availability of the specific component $(j)$, the connection availability of the total network can be shown with the Eq. 2.4.

$$
A_{i}=\prod_{j \in S_{i}} A_{j}
$$

If either of the connections of the primary path $(p)$ or backup path $(b)$ can be used, "1+1 protected" connection $(c)$ becomes the available state. The $c$ indicates the path as the backup purpose when the primary path fails. In short, the connection availability of the total network can show this with the Eq. 2.5.

$A_{e}=1-\left(1-A_{p}\right)\left(1-A_{b}\right)$

The $\boldsymbol{A}_{c}$ in the Eq. 2.5 is the item shown with the connection availability of the total network. The $\mathbf{1}-\boldsymbol{A}_{p}$ is the connection availability about the entire primary path. And the $\mathbf{1}-\boldsymbol{A}_{b}$ indicates the connection availability when the reserved backup path of the relevant primary path in advance operates in case the connection designated as the primary path fails.

There is the primary path as the appointed partner of the backup path in advance. At this time, the backup path has to select the route which any link doesn't overlap with the primary path. That is, the backup path exists independently with the primary path.

\section{Performance analysis of the proposed modeling}

In this chapter, the method getting the number of the nodes and links for comprising the logical topology of the light network was suggested through to interpret the definition of the availability of the optical network as the reverse. The procedure is comprised of 3 steps.

\section{Step1}

If the network is assumed as the logical topology of the full mesh type meaning the worst case, the number of the nodes and links are calculated in order to calculate the of node pairs according to the availabilities. The table 2 is the result.

Table 2 The number of the node pairs according to the target availabilities

\begin{tabular}{|l|c|c|}
\hline $\begin{array}{c}\text { Target } \\
\text { availabilities[\%] }\end{array}$ & $\begin{array}{l}\text { Number of the node pairs } \\
\text { in case of the single path }\end{array}$ & $\begin{array}{l}\text { Number of the node pairs in case } \\
\text { of the two path independent }\end{array}$ \\
\hline 99.5 & 55 & 55 \\
\hline 99.9 & 10 & 55 \\
\hline 99.95 & 0 & 55 \\
\hline 99.99 & 0 & 55 \\
\hline 99.995 & 0 & 55 \\
\hline 99.999 & 0 & 45 \\
\hline 99.9995 & 0 & 30 \\
\hline 99.9999 & 0 & 3 \\
\hline
\end{tabular}

In case of the single path among the item of the table 3.1, to get the number of the node pairs are implemented as the primary path. In conclusion, this means the degradation of the network reliability and availability. That is, if some availability is exceeded, it means that the network is unable to accept any more. 
And, in case of the two paths independent, it is designed considering the backup path in order to compare the primary path fails. In conclusion, it means to display the superior availability more than the case using one primary path.

\section{Step 2}

The method finding "the combination about the mutually different elements" of the statistics was used in order to calculate the number of nodes after finding the number of the node pairs. That is, each node is the combination about the node set which is distinguished and not overlapped. In this case, the number of combinations can be found by using the equation (3.1).

$$
{ }_{n} c_{r}=\frac{n !}{r !(n-r) !}
$$

In the Eq. 3.1, the $n$ means the number of possible all node pairs according to the network availability. And the $r$ means any pairs among all node pairs gotten as the result. That is, it becomes the concept which seems to get the number of specific node pairs in which the connection is made among the node pair of the $n$.

In conclusion, this node pair means the condition where it is being communicated through the positioned node and link in the center of the network which will be designed by the source transponder and destination transponder. At this time, the number of nodes are subtracted the source transponder and destination transponder from ${ }_{n} c_{r}$.

\section{Step 3}

It is calculated by using the gotten number of the nodes in the Step 2) that the number of the necessary links to complete the logical topology of the full mesh type. At this time, it is gotten by using the Eq. 3.2.

$$
l=\frac{n(n-1)}{2}
$$

In the Eq. 3.2, the $l$ means the number of the links, and the $n$ means the number of the nodes.

Table 3 The number of the nodes and node pairs

\begin{tabular}{|c|c|}
\hline Number of the node pairs & Number of the nodes \\
\hline 55 & 11 \\
\hline 45 & 10 \\
\hline 30 & 8 \\
\hline 3 & 3 \\
\hline
\end{tabular}

\section{Conclusion}

In this paper, we proposed a method to design the logical topology to reflect the characteristic of the optical network by using the availability model. The availability model of the optical network is the sum of the availability of a whole network when the source transponder and destination transponder are connected mutually. If a communication network is configured between the source and destination transponders by using the availability, the number of nodes and links can be calculated in the middle of the communication network. By using the availability model, one connection can be extended to the entire network and implemented to the logical topology of the optical network. In special, the availability is advantageous to the designer and operator of network because they can consider regional features if constructing network. 
On the other hand, the network operator or designer can determine various types and numbers of components according to the design costs, transmission QoS, and performance of network. In real, designing network has considerably limitation if considered just the primary and backup paths.

In conclusion, we proposed novel a method to construct an optical network by using availability model to accept additional nodes. If we design an optical network by using our novel method and by considering design and maintenance costs, we can select types of nodes and link components and configure efficient communication network.

\section{References}

[1] Donald Gross, Carl M. Harris, 1998, Fundamentals of Queueing Theory, Third Edition, John Wiley \& Sons, Inc.

[2] D. A. Schupke, "Availability-Based Service Providing in Optical Networks," IEEE Global Telecommunications Conference (GLOBECOM), Nov. 2005. Tutorial.

[3] James D. McCabe, 2003, Network Analysis, Architecture \& Design, Second Edition, Morgan Daufmann Publishers.

[4] Thomas G. Robertazzi, 1994, Computer Networks and Systems : Queueing Theory and Performance Evaluation, Second Edition, Springer-Verlag.

[5] J. Zhang, K. Zhu, B. Mukherjee, and H. Zang, "Service provisioning to provide per-connectionbased availability guarantee in WDM mesh networks, "Proceedings of the IEEE/OSA OFC, Mar. 2003. 